\title{
A Pet-raising Simulation Game using Multi-generations and Multi-scenarios
}

\author{
Kyungrok Byun and Haewon Byun* \\ Department of Convergence Security Engineering \\ *School of Information Technology \\ Sungshin Women's University, Korea \\ patricia97@naver.com,*hyewon@sungshin.ac.kr
}

\begin{abstract}
In this paper, we develop a pet-raising simulation game with multi-generations and multi-scenarios. For the multi-generations, we implement a genetic factor by using the Mendel's laws so as to carry recessive genetic traits including an external element and internal element from one generation to another generation. The external element is a pet appearance such as that of a pet head, body, face, ear and tail and the internal element is a pet ability. We also provide a user selection for the character customization. Both a genetic factor and user selection for the pet customization provides the infinite number of pet appearances theoretically. The result shows that raising a pet character with the alternation of generations affect players' affection to the game character and still players were interested in the alternation of generations itself. Also, the results showed that they could be interested in the games containing the multiple generations.
\end{abstract}

Keywords: game, pet-raising simulation game, character simulation game, multiple generations, multiple scenarios, genetic factor, character customization, appearance change

\section{Introduction}

Life simulation game is a genre that aims users' entertainment and fulfillment through the events which can make differences of the results according to the users' behavior [1,2]. Recently, numerous variations are being made due to the genres' popularity.

In the current existing life simulation games, the player had to be brought back to the point which the problem hasn't occurred yet, or had to play with the other game character which has no relevance with the pre-existing character when the problem occurred in the game due to the players' action or by the game system. However, both sides detract a game user's interest from the game character and the game itself. In the game 'Princess Maker', the player has to go back to the previously saved data or is burdened to raise the character which has grown differently from the player's intention as he or she desired. In Tamagotchi, this unintentional growth of the character has become a social issue, since the players starve their characters to death and give no medicine to kill, etc.

Unlike real-life pets, the next generation of the game characters can continue existing in the game. Thus, it can lessen the burden of losing the pet permanently. There are many people suffering from pet loss when they die or no longer exist. The same goes for games. Recently, there have been cases showing the possibility of empathy with pet robots, and moreover with virtual creatures. In comparison to this point, it appears that positive reactions can be made even if the pre-existing pet character is gone, and subsequent pet character appears.

Received (September 9, 2017), Review Result (December 2, 2017), Accepted (December 7, 2017)

* Corresponding Author 
According to Gi-Hyun Yang and Chul-ho Paik's research [3], the desire of customizing character's appearance affects users even during game play. Changing appearance during game play gives users positive game experiences. So a generational replacement function that can continue to control the appearance of the next game character can also provoke positive reactions to the game users.

In this paper, we proposed a simulation game using multi-generations and multiscenarios. We researches whether a positive reaction can be obtained from game users, by developing a game which a part of the game character succeeds to the next generation's character.

\section{Related Work}

Various researches have been conducted to stimulate game players' attention and interest by using game characters' appearance. Mi-sook Kim and Tae-won Kang's research [4] shows that using genetic algorithms can produce various types of game characters. After dividing the worm character with five chromosomes which correspond to the tail, body, face, eye, iris, they displayed the data in Structure types. The genetic operators showed diversity by using only mutations. In this paper, no genetic algorithm was used. Instead, each of the elements of the gene is divided into different parts of the body which have used string type, and changed them according to the laws of Mendel.

Seung Won Kwon and Gho Kim 's research[5] shows that the more players in the game are free of customizing character and a story in the game is richer, it has a positive impact on the player's gaming attitude and SCR(Skin Conductance Response). It is necessary to present a representative character that is continuously customizable because it needs to be more interesting and entertaining. In this paper, a chimera character was produced as a representative character.

In addition, according to research by Shin Dae-young[6], the 2010 edition of the South Korea's Game White Paper suggests that although the popularity of game characters is not much of a factor in the popularity of gaming, but the importance of the character can be realized through many antecedent studies.

There were also games like 'Ovipets[7]', which used genetic factors, or 'TamagotchI[8]' which led a generation from parent to offspring. However, it is difficult to know whether the genetic factor has a positive effect on raising game character, since it is the only factor that the raising is feeding. In Tamagotchi, raising current game character does not affect the ability of the next generation [9].

In this paper, it applied inheritance appearance change into the game and factors that current character's status which affects to the maximum status of future generations. Additionally, players can choose their character's partial appearance which allows customization to be more freely.

\section{Implementation}

\subsection{Game Planning}

The design of this game was conducted by reference of the game plan standard form which was published by the Korea Creative Content Agency.

(1) Idea generation

It is the first step in decision-making when developing a game. It is a form of ideas suggesting the direction of the most basic of the game. 
(2) Game background storyline

It has written about a comprehensive view of the game, character set up, and concepts. It's format is character related settings document for common concept design.

(3) Create rules for games

Rules were specified for raising characters and the generation change in the game. It belongs to a common detailed design step.

(4) Run prototype

A prototype has been made to model the appearance of a game character changes randomly when generations goes to next one. Indeed, it is confirmed that the appearance of the character has changed.

(5) Detailed rule confirmation

Since then, partial customization factors and genetic factors were inserted into the game. If the player chooses the desired part of game character, the part will be inherited as it is.

(6) Development and modification

After running prototypes, added and modified the rule's detail to suit the game.

(7) Beta test after completion

We checked the completeness of the game and the character generation replacement through beta testing.

\subsection{Prototype Result and Planning Modification}

The design of this game was conducted by reference of the game plan standard form which was published by the Korea Creative Content Agency. The first prototype did not apply to genetic rules to the next generation. Instead, it applied the appearance of the current character and the partner character randomly. As shown in Table 1, results of running prototypes 5 times ensure that different outcomes are shown between 1 st and $3 \mathrm{rd}$ generations.

After running the prototype, projects have been partially modified. Instead of succeeding appearance randomly to the next generation, it follows general genetic laws and added some appearance parts to the game for more diversity. The 1st generation, 5th generation, and 10th generation were compared for samples that applied genetic factors. Again, we could see that different outcomes were derived from first-generation and future generations.

\section{Table 1. Differences of the Game Characters Appearance by Generation}

\begin{tabular}{|c|c|c|c|}
\hline No.1 & 1st Generation & 2nd Generation & 3rd Generation \\
\hline Head & Gray/Short Fur & White/Long Fur & Yellow/Short Fur \\
\hline Body & Gray/Short-Coat & Gray/Medium-Coat & Gray/Medium-Coat \\
\hline Face & Green-Eyed/Round & Green-Eyed/Round & Green-Eyed/Round \\
\hline Ear & Gray/Lop-eared & Gray/Ears Up & Gray/Ears Up \\
\hline Tail & Gray & Gray & Gray \\
\hline No.2 & 1st Generation & 2nd Generation & 3rd Generation \\
\hline Head & Yellow/Short Fur & Yellow/Long Fur & White/Long Fur \\
\hline Body & Yellow/Short-Coat & Yellow/Medium- & Yellow/Medium- \\
\hline Face & Blue-Eyed/Round & Blue-Eyed/Round & Blue-Eyed/Round \\
\hline Ear & Yellow/Lop-eared & Yellow/Lop-eared & Gray/Lop-eared \\
\hline Tail & Yellow & Yellow & Yellow \\
\hline
\end{tabular}




\begin{tabular}{|c|c|c|c|}
\hline No.3 & 1st Generation & 2nd Generation & 3rd Generation \\
\hline Head & Gray/Short Fur & Gray/Short Fur & Gray/Short Fur \\
\hline Body & Gray/Short-Coat & Gray/Short-Coat & Gray/Short-Coat \\
\hline Face & Green-Eyed/Round & Green-Eyed/Round & Green-Eyed/Round \\
\hline Ear & Gray/Lop-eared & Yellow/Lop-eared & Yellow/Lop-eared \\
\hline Tail & Gray & Gray & Gray \\
\hline No.4 & 1st Generation & 2nd Generation & 3rd Generation \\
\hline Head & Yellow/Short Fur & Gray/Short Fur & Gray/Short Fur \\
\hline Body & Yellow/Short-Coat & Yellow/Short-Coat & Yellow/Short-Coat \\
\hline Face & Blue-Eyed/Round & Blue-Eyed/Round & Blue-Eyed/Round \\
\hline Ear & Yellow/Lop-eared & Gray/Lop-eared & Gray/Lop-eared \\
\hline Tail & Yellow & Gray & Gray \\
\hline No.5 & 1st Generation & 2nd Generation & 3rd Generation \\
\hline Head & Yellow/Long Fur & Yellow/Long Fur & Yellow/Long Fur \\
\hline Body & Yellow/Medium- & Yellow/Medium- & Gray/Medium-Coat \\
\hline Face & Purple-Eyed/Round & Blue-Eyed/Round & Pink-Eyed/Sharp \\
\hline Ear & Yellow/Ears Up & Yellow/Lop-eared & Yellow/Lop-eared \\
\hline Tail & Yellow & Yellow & Yellow \\
\hline
\end{tabular}

Table 2. Differences of the Game Characters Appearance by Generation with Heredity

\begin{tabular}{|c|c|c|c|}
\hline No.1 & 1st Generation & 5th Generation & 10th Generation \\
\hline Head & Gray/Short Fur & Yellow/Short Fur & Yellow/Long Fur \\
\hline Body & Gray/Short-Coat & Yellow/Long-Coat & Yellow/Medium- \\
\hline Face & Green- & Purple-Eyed/Anime & Blue-Eyed/Round \\
\hline Ear & Gray/Lop-eared & Yellow/Lop-eared & Yellow/Lop-eared \\
\hline Tail & Gray & Gray & Gray \\
\hline No.2 & 1st Generation & 5th Generation & 10th Generation \\
\hline Head & Yellow/Short Fur & Yellow/Short Fur & Gray/Short Fur \\
\hline Body & Yellow/Short-Coat & Yellow/Short-Coat & Yellow/Long-Coat \\
\hline Face & Blue-Eyed/Round & Purple-Eyed/Anime & Red-Eyed/Anime \\
\hline Ear & Yellow/Lop-eared & Yellow/Lop-eared & Yellow/Lop-eared \\
\hline Tail & Yellow & Yellow & Yellow \\
\hline No.3 & 1st Generation & 5th Generation & 10th Generation \\
\hline Head & Yellow/Short Fur & Yellow/Short Fur & Gray/Short Fur \\
\hline Body & Yellow/Short-Coat & Yellow/Long-Coat & Stripe/Yellow/ \\
\hline Face & Blue-Eyed/Round & Purple-Eyed/Anime & Purple-Eyed/Anime \\
\hline Ear & Yellow/Lop-eared & Yellow/Lop-eared & Yellow/Lop-eared \\
\hline Tail & Yellow & Yellow & Yellow \\
\hline No.4 & 1st Generation & 5th Generation & 10th Generation \\
\hline Head & Gray/Short Fur & Gray/Short Fur & Yellow/Long Fur \\
\hline Body & Gray/Short-Coat & Yellow/Medium- & Stripe/Yellow/ \\
\hline Face & Green- & Green-Eyed/Round & Green-Eyed/Round \\
\hline Ear & Gray/Lop-eared & Yellow/Lop-eared & Yellow/Lop-eared \\
\hline Tail & Gray & Yellow & Yellow \\
\hline No.5 & 1 1st Generation & 5th Generation & 10th Generation \\
\hline Head & Gray/Short Fur & Gray/Short Fur & Yellow/Short Fur \\
\hline Body & Gray/Short-Coat & Yellow/Short-Coat & Yellow/Long-Coat \\
\hline Face & Green- & Red-Eyed/Anime & Green-Eyed/Round \\
\hline Ear & Gray/Lop-eared & Yellow/Lop-eared & Yellow/Lop-eared \\
\hline Tail & Gray & Yellow & Yellow \\
\hline & & & \\
\hline
\end{tabular}




\subsection{Game Development}

In this paper, we developed a multi-scenario $2 \mathrm{~d}$ game using Unity engine. The goal is to join a virtual company and develop diversified types of chimera. On a certain days, events related to that date appear in the Adventure dialog format. 4 status values have been set to make changes to the event content.
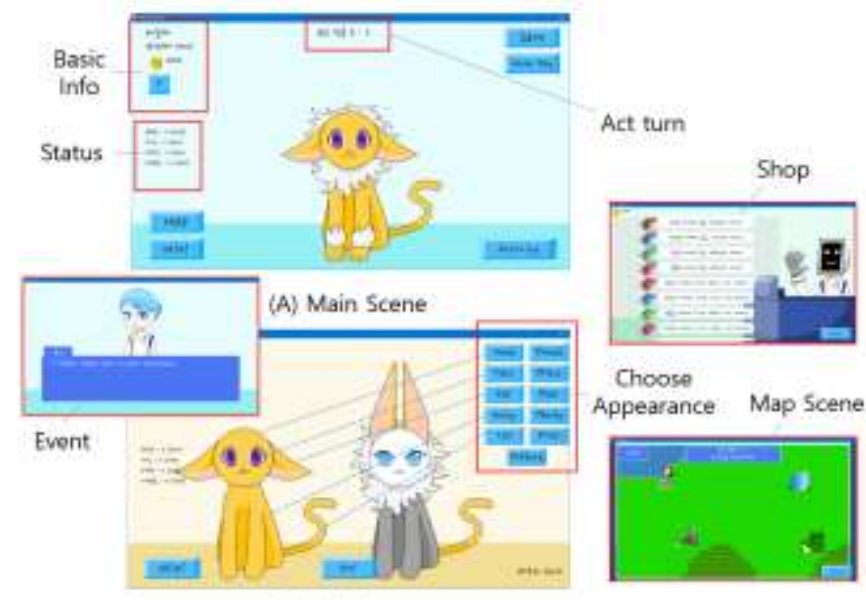

(B) Mix Scene

Figure 1. Game Prototype

\subsubsection{Game Overview}

This game uses the status of the game character for multi-scenario, and has the following game flow. Player can choose daily activity or career activity in a room. Game characters show different responses in the event of the lapse of time. In this paper, various texts will be printed in the event depending on the player character's status which the player has raised. Therefore, players can verify different events result from each generation.

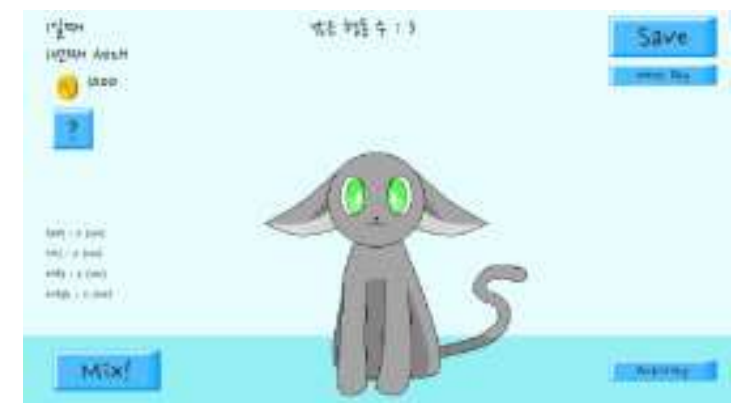

Figure 2. Main Scene Example

Simulation games amuse players by giving different reactions depending on which direction they develop characters. In this paper, 4 status points(stamina, knowledge, charm, and affection) were placed in the corresponding game. It is that stamina, knowledge, attraction, and affection. Increasing the number of the status will result the character enables to do business activities, which depends on status the player increased.

As shown in Figure 3, the status of game character increases when doing daily activities, and the status constitutes a condition for business activities to earn in game money. Work activities cannot be carried out unless more than a certain level of status is attained. The in game money will be used to purchase items and increase their status to 
higher capacities. Depending on which status the player has raised, the max status of the next generation increases correspondingly.

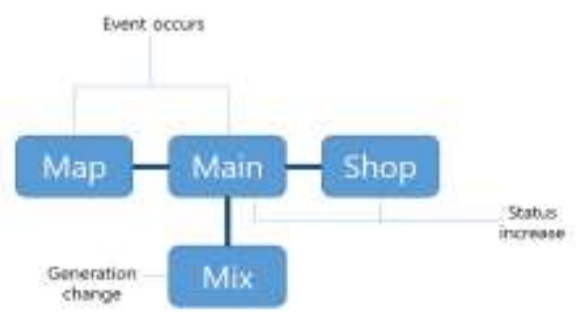

Figure 3. Game Structure

\subsubsection{Game Flow}

When player starts a game, tutorial shows various explanations in the form of text adventure games. In this paper, the player will raise a randomly generated chimera. Chimera, which is intended to be raised is randomly generated at start, but player can change some of its appearance through generational replacement. The maximum status of the next game character is increased through changing generations of game characters. In this process, players are imposed to custom factors and gene factors that allow players to select parts of their character's appearance during a generational change in order to relieve their boredom. After the generation change, newly created character will be raised.

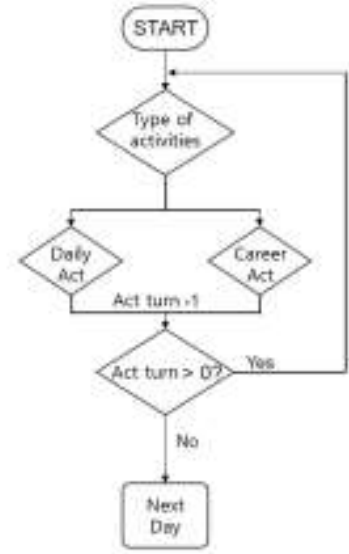

Figure 4. Game Flow

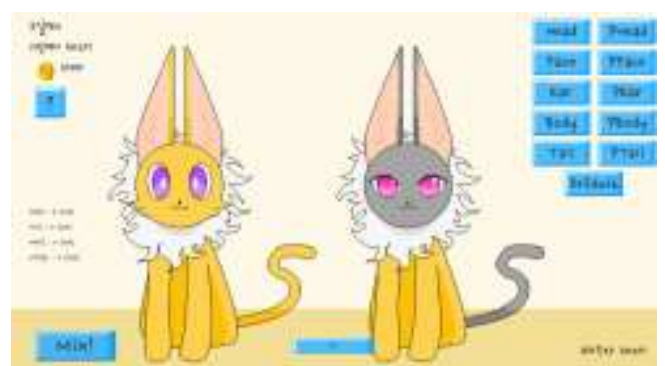

Figure 5. Generation Change Scene Example

\subsubsection{Scenario Factor}

\section{Leftover act turn}

A act turn decreases one by one if player earn money by making character work, increasing character's abilities, etc. The act turn specified per day is 3. Every time when player moves to the next date, player get 3 new act turns. When the act turn is 0 , no other action can be done except purchasing item from the store and going over to the next day.

\section{Date and generation}

It is designed to view different events according to the period of game play. A game character status checking event takes place once every 10 days. A contest event will be held for awarding prizes based on the status of the characters once every 30 days. Current generation increases when player changes current game character. The date and generation are located at the top left of the main game screen and can be checked anytime. 


\section{Item}

Items can be bought at shop and the character's status will be increased according to the item which has been purchased. There are currently 8 kinds of items at shop and they can be used to increase the ability to match the corresponding status by 50 to 100 . Also, items are used concurrently with purchase.

\section{Appearance Changes}

The appearance of character is divided into parts with head, torso, tail, ears, and face. Each part has its own set of gene strings which determines the appearance of the offspring. Players can choose one of the parts from current character or randomly generated partner characters, such as [Fig.4], if they want when replacing the generation. The next generation character will maintain the same appearance of the applied part and same gene strings. If the player chooses nothing, then each part follows a genetic string combination of the existing character and partner character that leads to the next generation. The genetic string combination follows the laws of Mendel.

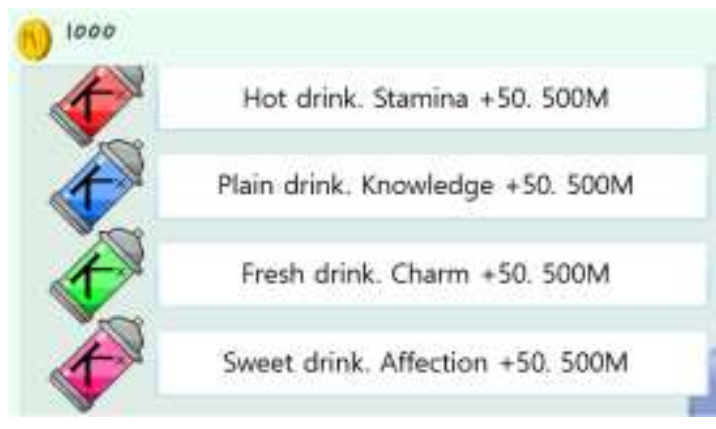

Figure 6. Game Items

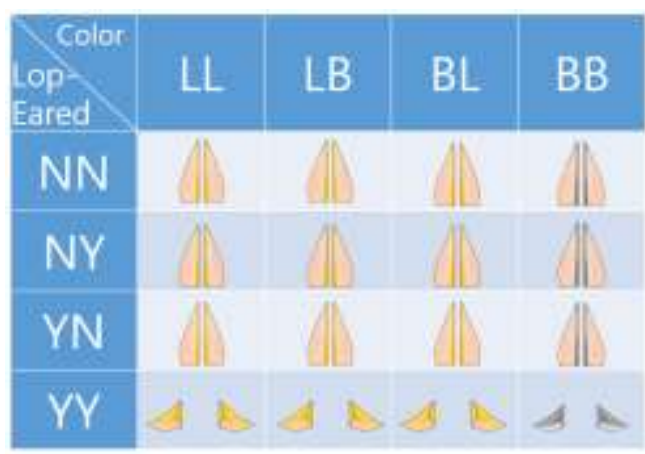

Figure 7. Game Character's Ears By Gene String

\section{Experimental Results}

In our experiments, three users were playing the game for collecting the data for the appearance changes of game character. We recorded the appearance changes by only a genetic factor from the first generation to the tenth generation. We do not allow the game users to select the character appearance option for speeding up the experiments. For the question investigation, the 7 game users raised the game characters from the first generation to the third generation in the 30 days by the game time. We made a shift in generations every 10 days and recorded the maximum ability and appearances of the character in each generation.

In the game survey, game users score 1 (not at all) to 5(absolutely) for the question items as follows. 


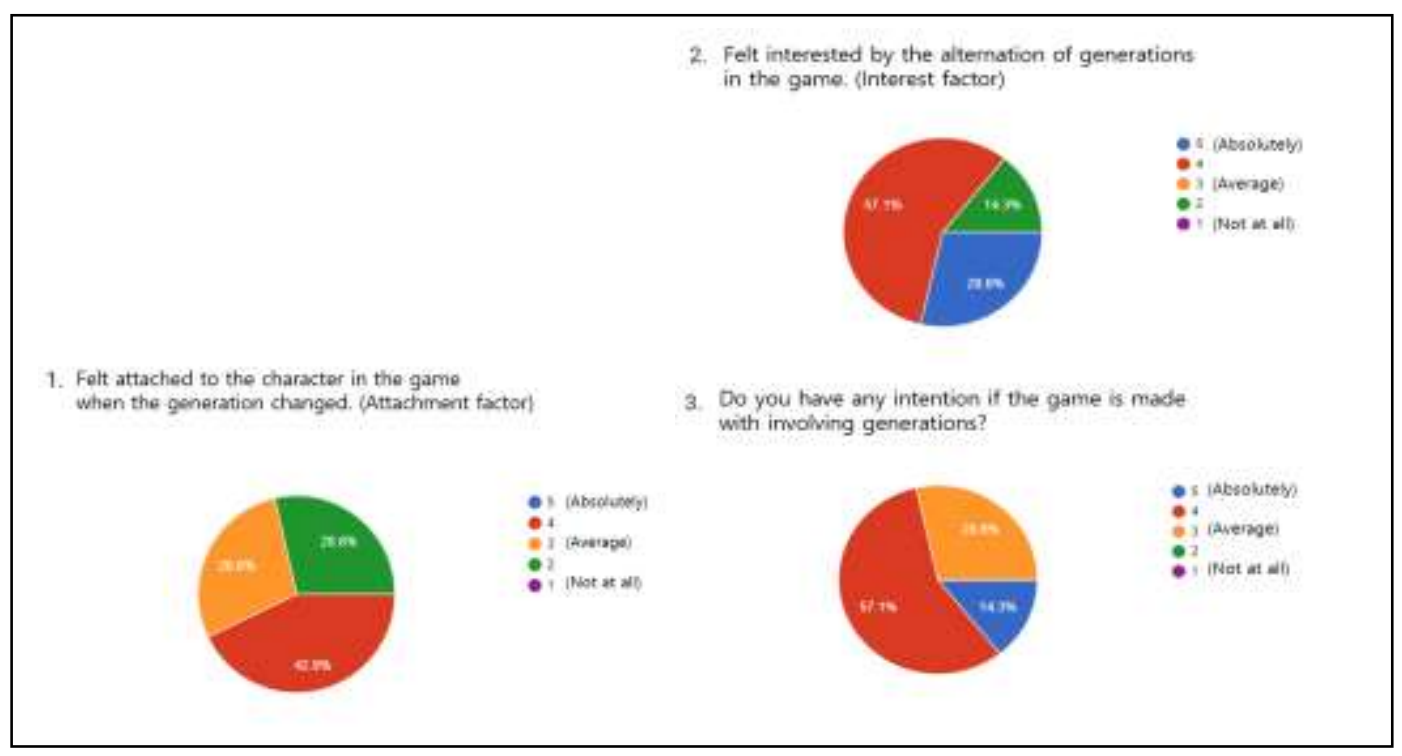

1. I felt attached to the game character when the generation changed. (attachment factor)

2. I felt interested by the alterations of generations in the game. (interest factor)

3. Do you have any intention if the game is made with involving generation changes ?

In the Table 3, we can see the customization results of the pet appearances. By using only genetic factors, we showed the various pet appearances. In the case of No.1 and No.2, there are 2 cases with the same pet appearances, but in the case of No.3, there are no duplications among the various pet appearances. By using both the genetic factors and user's selection for pet appearances, we can customize the infinite numbers of pet appearances theoretically.

In the Table 4, the game surveys collect user's evaluation of our game. The average of the answer to the first question is 3.15 and the average of the answer to the question 2 is 4 and the average of the answer to the question 3 is 3.86 . The truncation of the float average values is made to 3 digits as 0.001 . It means that the game users felt attachment more or less to the pet after the generation changes and users were interested in the generation changes of the game. If there is another game with the factors of generation changes, game users mentioned that they would want to play the games.

\section{Table 3. Various Game Character Appearances from the First Generation to The Tenth Generation}

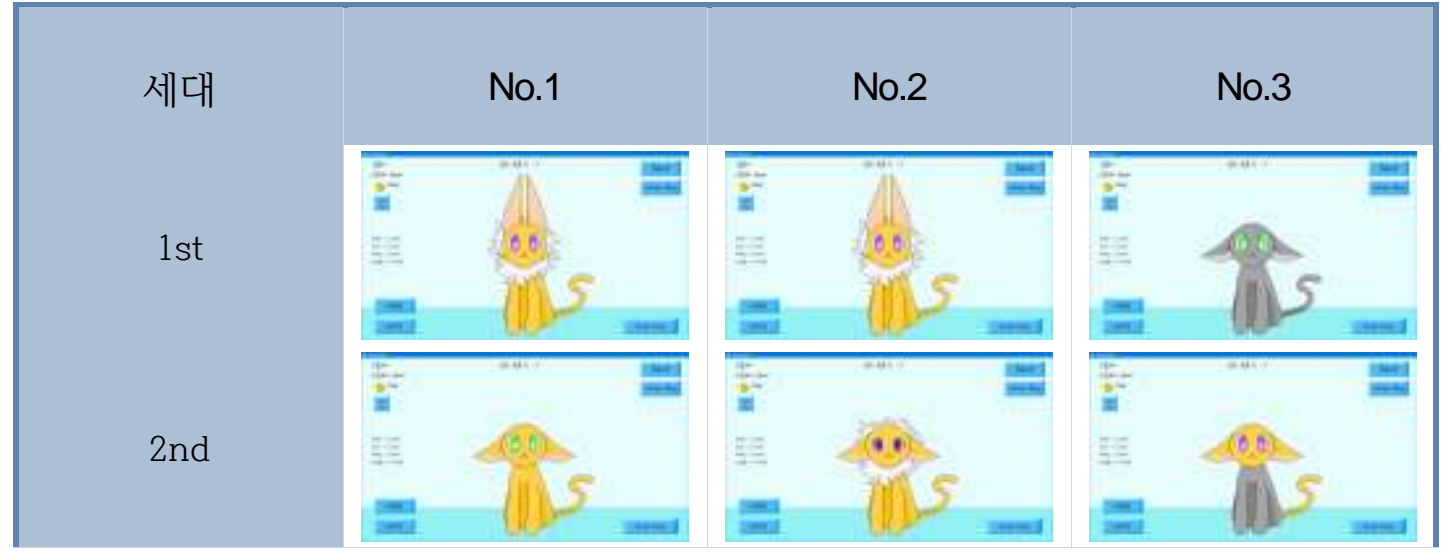




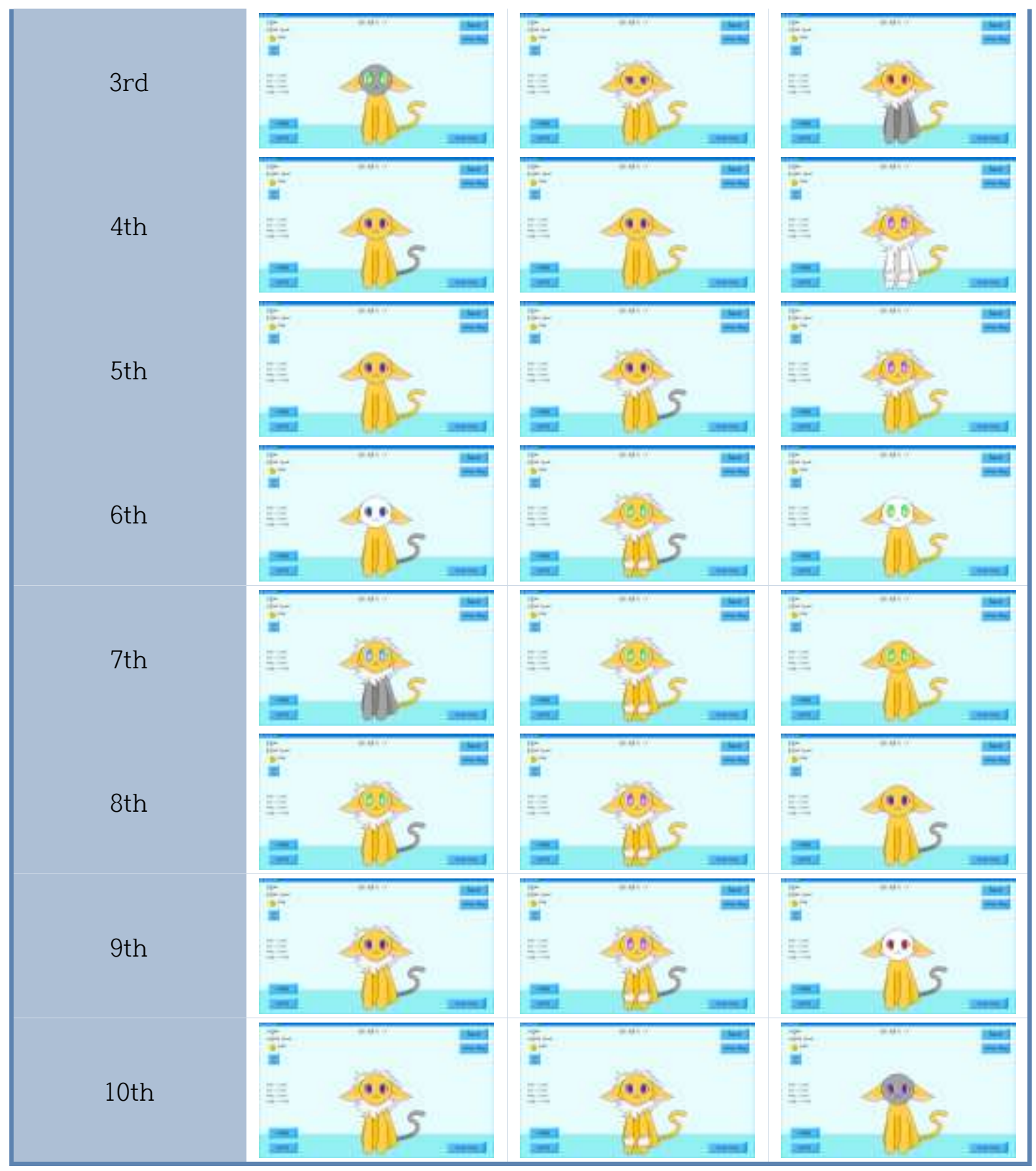

\section{Conclusion}

In this paper, we proposed the skim for the generation changes and multiple scenarios on the pet-raising simulation game to increase the game user interest. We planned the game by following the standard documents of game plan and made a game with generation changes and multi-scenarios. To show the effectiveness of our game, we measured on how the appearance changes of a pet from generation to generation affect game user interests.

Theoretically, we showed we can make the infinite number of the various appearances for the pet on our pet-raising simulation game. The infinite number of the various appearances for the pet is important to encourage game users playing and to enjoy the game. The game surveys presented that the game users felt attachments to the characters with generation changes and they would like to play another character simulation game with the concept of multiple generations. 


\title{
Acknowledgements
}

This work was supported by the Sungshin Women's University Research Grant of 2017.

\section{References}

[1] D.-H. Lee, "The whirl of fashion 'Social·Simulation·War' games are popular", Digital Daily, http://www.ddaily.co.kr/news/article.html?no=146742, (2016).

[2] E.-J. Park, "In Japan, a Sony robot dog called 'aibo"s funeral was held", Korea Joongang Daily, http://news.joins.com/article/17250097, (2015).

[3] G.-H. Yang and C.-H. Paik, "A Study on the User Immersion and Loyalty of MMORPG Avatar Customization", Journal of Korea Game Society, vol.13, no.2, (2013), pp.17-28.

[4] M.-S.Kim and T.-W. Kang, "Evolutionary Design of Game Character", Workshop presentation file of Korean Institute of Information Scientists and Engineers, spring, vol. 30, no. 1(B), (2003), pp.410-412.

[5] S. W. Kwon and G. Kim, "Effects of Game Character Customizing and Game Story on MMORPG Game Attitude and Skin Conductance Response”, Journal of Consumer Studies, vol. 25, no. 3, (2014), pp.21-43.

[6] D.-Y. Shin, "Relation of Game User and Game Character", Journal of Korea Society of Computer Information, Winter Conference, vol. 21, no. 1, (2013), pp.51-52.

[7] IOHAZE, "Ovipets", from http://ovipets.com/, (2010).

[8] BANDAI NAMCO Entertainment Inc., "Tamagotchi”, from http://tamagotch.channel.or.jp/index.php, (1996).

[9] Y. R. Lee and Y. Kim, "Implementation and Evaluation of An Open Scenario Online Horror Game to Produce Infinite User Experiences”, Journal of The Korean Society for Computer Game, vol. 27, no. 4, (2014).

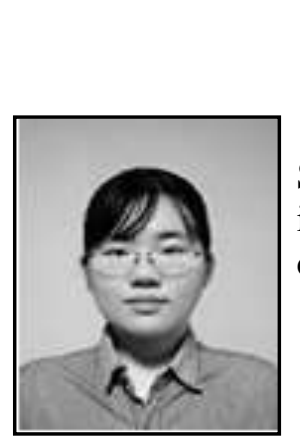

\begin{abstract}
Authors
Kyung-Rok Byun, she is a senior in Department of Convergence Security Engineering of Sungshin Women's University. Her research interests include game, computer graphics, virtual reality and block chain algorithm.
\end{abstract}

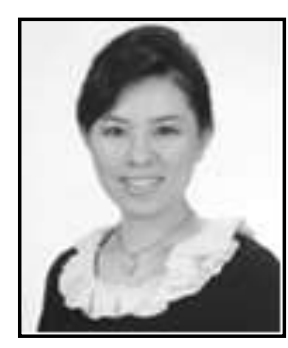

Hae-Won Byun, she is a professor in School of Information Technology of Sungshin Women's University. Her research interests include computer graphics, virtual reality and big data analysis. 